\title{
Neuraltherapie bei Nacken- und Spannungskopfschmerz
}

Autor: Uwe Günter

\section{ZUSAMMENFASSUNG}

Die Neuraltherapie ist als diagnostische und therapeutische Anwendung von Lokalanästhetika beim chronisch-rezidivierenden Nacken- und Spannungskopfschmerz adjuvant einsetzbar, risikoarm, kostengünstig und erfolgreich.

Diese Injektionen werden im Bereich eines jeden schmerzhaften Dermatoms, verquollenen Subkutoms mit Verbindungen zum perivaskulären Sympathikus, druckdolenten Faszio-Myotoms (z. B. Triggerpunkt), an Nerven und Ganglien des Grenzstrang unter standardisiertem Knochenkontakt mit und ohne Sonographie-Monitoring sowie an Störfeldern wie Tonsillen, Sinus, Zähnen usw. durchgeführt und kann jeder Arzt erlernen bzw. ambulant und stationär anwenden.

Das Ester Procain ist trotz des Überwiegens der Studien mit Lidocain u. a. Amiden nach persönlichem Erfahrungsaustausch mit seit mehr als 50 Jahren praktisch tätigen Autoren und aktuellsten Literaturdaten überlegen bzw. am besten zu überwachen.

Schlüsselwörter

Chronisch-rezidivierender Nackenschmerz, Spannungs-

kopfschmerz, temporomandibuläre Biss-Relationsstörung, Neuraltherapie, Lokalanästhetika, Procain

\section{ABSTRACT}

Neural therapy can be adjuvantly used as a diagnostic and therapeutic application of local anesthetics for chronic-recurrent neck pain and tension headache, low-risk, cost-effective and successful.

These injections are made in the area of each painful dermatome, swollen subcutaneous tissue with connection of the perivascular sympathetic, pressure-thalid fascio-myotome (e.g. trigger point), to nerves and ganglia of the sympathetic trunk under standardized bone contact with and without sonography monitoring as well as to interference fields such as tonsils, sinuses, teeth etc. performed and can be learned by every doctor or used on an outpatient and inpatient basis.

The ester procaine, despite the preponderance of studies with lidocaine et al. amides after personal exchange of experience with more than 50-years-practical-active authors and most recent literature data superior or best to monitor.

Keywords

Chronic-recurrent neck pain, tension headache, temporomandibular disorder, neural therapy, local anesthetics, procaine

\section{Einleitung}

Der Nackenschmerz projiziert sich als unspezifisches Zervikalsyndrom auf die Dermatome unterhalb der Linea nuchae und oberhalb des Dorns Th1 zwischen den Schulteransätzen des M. trapezius [1]. Nach Hansen und Schliack kann man von einer Projektion in die dorsalen Anteile von C3 und C4 ausgehen [2].

Beim Spannungskopfschmerz projiziert sich der Schmerz hauptsächlich auf das Dermatom C2 sowie in den Bereich des 1. Trigeminusastes [2].

\section{Prävalenz}

\section{Nackenschmerz}

Die Ein-Jahres-Prävalenz von isolierten Nackenschmerzen wird 2015 mit mehr als $56 \%$ und bei zusätzlichem Kopfschmerz mit ca. $85 \%$ angegeben [3].

Die Deutsche Gesellschaft für Allgemein- und Familienmedizin (DEGAM) geht von einer Langzeitprävalenz des Nackenschmerzes von ca. $50 \%$ aus [5].

\section{Spannungskopfschmerz}

Göbel gibt die Prävalenz von meist nuchal beginnenden und zu ca. $44 \%$ beidseits auftretenden Spannungskopf- schmerzen mit über $70 \%$ an [6], was die Akershus-Studie bestätigt [7][8].

\section{Ätiopathogenese und Risiken}

\section{Nackenschmerz}

In der Ätiologie der Nackenschmerzen werden neben den radiologischen Kausalitäten Einflussfaktoren wie Übergewicht, Schwangerschaft, starke körperliche Arbeit, Lebensalter, subjektive Gesundheitseinstellung und chronischer Stress angegeben [4][5].

Seidel fasst 2015 nach einer Originalpublikation von Hansraj den Einfluss vom Smartphone auf die HWS zusammen [9].

Weinschenk und Mitarbeiter gehen beim chronischen Nackenschmerz davon aus, dass es durch neuroanatomische Beziehungen bei pharyngealen Erkrankungen zu dolenten Druckpunkten im Bereich des Trapezmuskels kommen kann, welche Gleditsch sowie Hülse und Kollegen seit Jahren im Zusammenhang mit HNO-Erkrankungen empirisch, anatomisch und pathophysiologisch beschreiben [10][11][12]. 
Bei eigenen Patienten sind neben diesen sinupharyngealen Beziehungen in der Anamnese Zystopathien, Störungen der Nierenfunktion, ein Nikotinabusus sowie eine Pathologie des Homocysteinstoffwechsels zu beobachten.

\section{Spannungskopfschmerz}

Beim Spannungskopfschmerz werden als Risiken Medikamentenübergebrauch, bio-psycho-sozialer Stress, Angstund depressive Störungen und von Patienten nervale, psychische und vegetative Störungen bzw. Erkrankungen der Zähne und Sinnesorgane angegeben [6].

In der Akershus-Studie sah man gehäuft jüngere Frauen sowie Komorbiditäten wie Erschöpfungs- und Müdigkeitssymptome [7][8].

In der Pathogenese werden eine Aktivierung von NMDARezeptoren mit steriler Entzündungsreaktion der C-Fasern und Mikroläsionen der Kopf-, Hals- und Kaumuskulatur favorisiert [6][7][13][14].

Vor allem bei der oromandibulären bzw. Bissrelationsstörung spielen pathologische Aktivitäten der Muskeln [6] [13][14] mit nachweisbaren EMG-Veränderungen, eine Dysbalance des Serotonin- sowie Endorphinspiegels eine Rolle [6].

Bei eigenen Patienten sind hierzu in der Anamnese bruxistische Stressreaktionen und Hinweise für erschöpfende Störungen bis hin zu Fatigue zu beobachten.

Eine Sonderform stellt der zervikogene Kopfschmerz als Dysbalance der oberen 3 zervikalen Segmente bzw. subokzipitalen Muskeln mit unilateralem nach temporal ausstrahlenden Referred Pain dar [15].

Hierbei treten nicht nur über die aus der Ansa cervicalis hervorgehenden $\mathrm{Nn}$. occipitales Schmerzen, sondern auch aufgrund der hochsensiblen neuroanatomischen Verschaltung dieser Segmente mit den Vagus- und Trigeminuskernen über die Formatio reticularis vegetative und trigeminale Symptome auf [12].

\section{Übersicht zu evidenten Therapiestrategien}

\section{Nackenschmerz}

Zu Nackenschmerzen empfiehlt die Leitlinie der DEGAM keine Medikation, dagegen physikalische bzw. Muskelkräftigungstherapien, Akupunktur sowie Injektionen in myofasziale Triggerpunkte, deren Wirksamkeit in Interventionsstudien belegt wurde [5].

Andere Fachgebiete gehen aktuell von einer multifaktoriellen Genese, jedoch individuelleren Therapiestrategien aus [16].

\section{Spannungskopfschmerz}

Zu Kopfschmerzen und Migräne geht aus der Leitlinie der Deutschen Gesellschaft für Neurologie (DGN) hervor, dass EMG-basierte Biofeedback-Therapie wirksam ist, Entspannungstechniken sehr wahrscheinlich wirksam sind, Physiotherapie, medizinische Trainingstherapie und Manualtherapie wahrscheinlich wirksam sind und es für die klassische Akupunktur eine geringe Evidenz für deren Wirksamkeit gibt [17]. Dagegen ergeben Studienanalysen von Akupunktur bei Kopfschmerz mehrfach signifikant positive Ergebnisse [18].

Die Kombination aus pharmakologischer Therapie und Stressbewältigungstraining ist erfolgreicher als die Einzeltherapien mit Analgetika oder Antidepressiva [17]. Göbel empfiehlt zusätzlich eine sogenannte Münz-Massage (Gua Sha) mit Pfefferminzöl und die Therapie einer oromandibulären Störung [6].

\section{Neuraltherapie}

\section{Grundlagen}

Zur multimodalen Therapie chronischer Nacken- und Spannungskopfschmerzen gehört auch die Neuraltherapie als minimalinvasives Verfahren. Darunter wird die diagnostische und therapeutische Anwendung von Lokalanästhetika im Segment, lokoregional und an Störfeldern verstanden [19][20].

Seit den Veröffentlichungen von Schleich, Spiess u. a. über die Anwendung von Lokalanästhetika bei Entzündungen und der Gebrüder Huneke über die Fernwirkung von Procain fasst die Neuraltherapie Weiterentwicklungen zahlreicher Injektionstechniken zusammen und kann auf wissenschaftliche Erkenntnisse über sogenannte alternative Wirkungen aller immunmodulativen Lokalanästhetika am G-Protein-gekoppelten Zellrezeptor aufbauen [19].

Die oft nur als „Quaddeln“ bekannte Methode ist retrospektiv eine häufig angewendete und erfolgreiche Therapie im Praxisalltag [21].

Unter den seit Jahrzehnten genutzten Mitteln liegen die Vorteile klar beim Ester Procain [22][23][24] und seinen durch die ubiquitär wirkende Pseudocholinesterase entstehenden Spaltprodukte Paraaminobenzoesäure und Diethylaminoethanol. Sie wirken u. a. vasodilatatorisch und positiv auf den Endocannabinoidspiegel [19].

\section{Therapeutisches Vorgehen beim chronischen Nacken- bzw. Kopfschmerz} Segmenttherapie

Nach Ausschluss von Organpathologien [4], entsprechender schriftlicher Aufklärung und analog der von Wander 


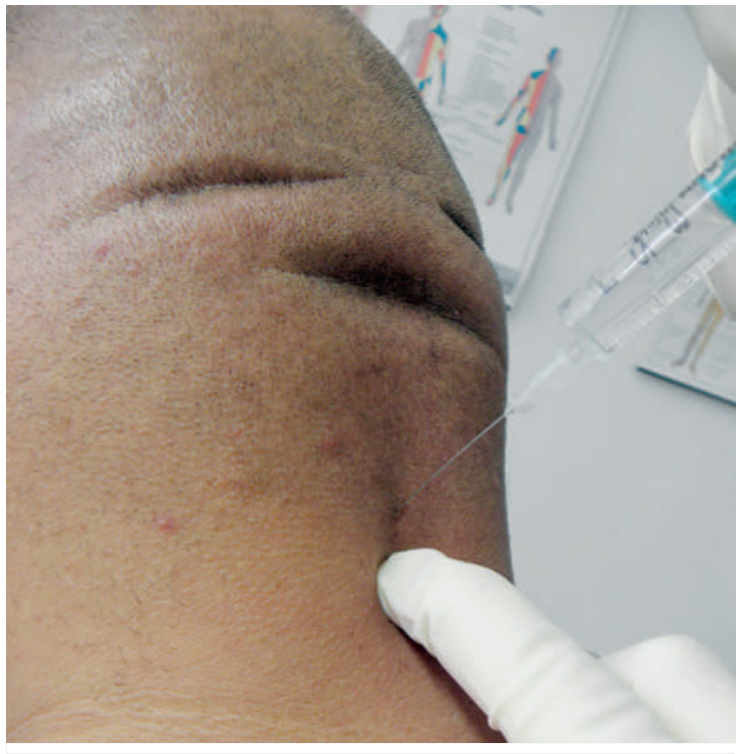

Abb. 1 Beginn der Injektion am Dorn C2. Foto: U. Günter

bereits 1992 sowie 2014 und des Autors 2018 bei der Neuraltherapie des Rückenschmerzes beschriebenen Vorgehensweise [25][26][27] beginne ich als Erstes mit intrakutanen Injektionen im Bereich der Dermatome C2-C4 und des 1. Trigeminusastes („Quaddeln“). Je nach Schmerztopographie (der Patient wird immer gebeten, die Beschwerden mit der Hand zu lokalisieren) können Injektionen an die Akupunkturpunkte Bla2-11, ExKH3, Ga1-21, 3E21-23, Dü2 sowie LG20 und ggf. ExKH1 (siehe Lehrbücher der Akupunktur) als Injektions- bzw. Neuralakupunktur folgen.

Die subkutane Therapie eines vorhandenen Lymphbelts [11] kann mit der präperiostalen Injektion an Schädelknochen und deren Suturae je nach Dolenz oder osteopathischer Läsion sowie an den tastbaren Dorn C2 kombiniert werden ( $\bullet$ Abb. 1). Obligat empfehle ich die Injektion an den Dorn C7, wobei wie bei der Technik am Dorn C2 auf die Stichrichtung und den Knochenkontakt geachtet werden sollte (Stichrichtung der Kanüle möglichst $90^{\circ}$ zur Dorntopographie). Durch infiltrierendes Vorgehen am Dorn C7 werden mit einer Injektion mehrere Segmente über ihre Untereinheiten (hier Dermatom C4, Subkutom C5, Fasziotom und Myotom des Trapezius, welcher bis zum Dorn des BWK 12 reicht, und damit C2-4 sowie das Sklerotom bzw. Osteotom C7) regulativ beeinflusst, was in der Akupunktur als Wirkung des Punktes LG14 („Spinne“) bekannt ist ( $\bullet$ Abb. 2).

Die Segmenttherapie wird je nach Anamnese und manuellem Befund in Folgesitzungen alle 7-28 Tage durch die hochwirksame Therapie vorhandener Triggerpunkte ( $\triangleright$ Abb. 3), wie sie u. a. durch Travell und Kollegen bereits 1942 veröffentlicht wurde und in der Akupunktur als Dry Needling erfolgreich angewendet wird, vervollständigt [28]. Aufgrund der vegetativen Reaktionen empfiehlt

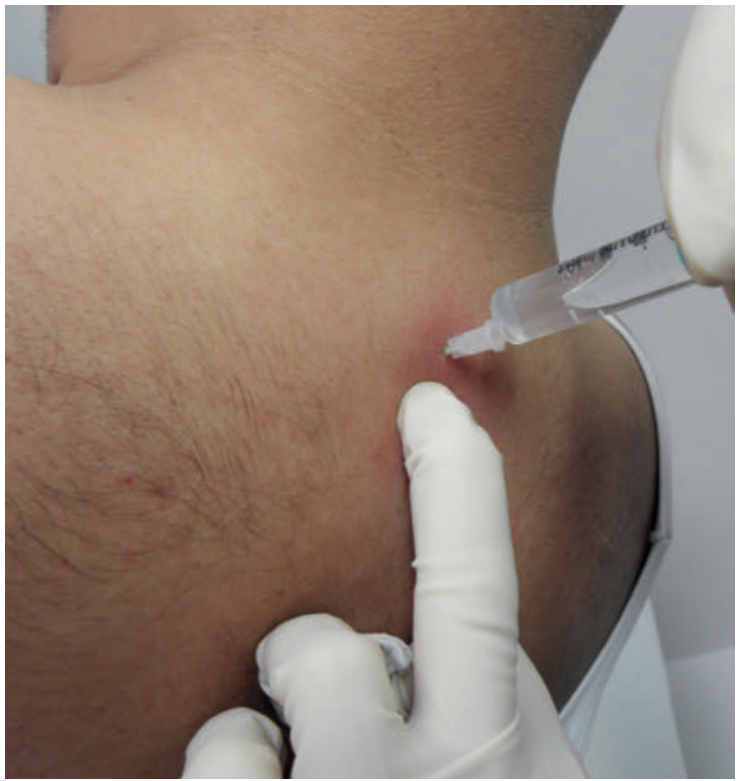

- Abb. 2 Injektion an Dorn C7. Foto: U. Günter

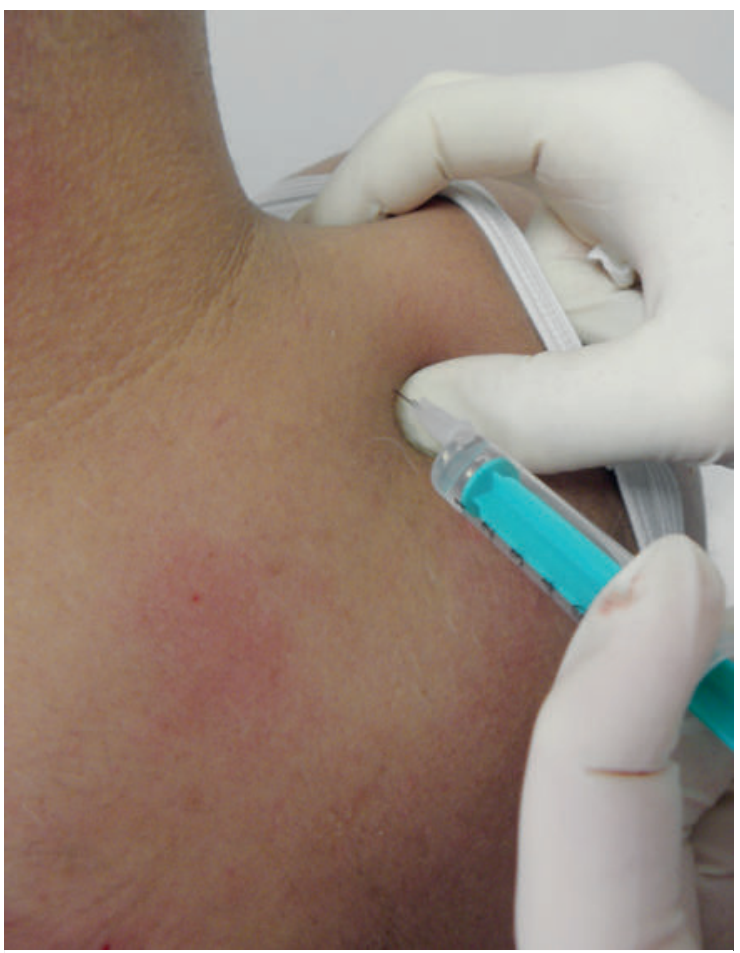

Abb. 3 Injektion in einen Triggerpunkt. Foto: U. Günter

sich nicht nur die Kontrolle der vom M. trapezius beeinflussten Wirbelsegmente vom Okziput bis Th12, sondern auch die Kontrolle der Herzratenvariabilität (HRV), des Pulses und des arteriellen Blutdrucks [29]. Unmittelbar post injectionem ist die postisometrische Relaxation (PIR) auch der anderen Muskeln (z. B. Mm. rhomboidei, levator scapulae sowie erector spinae) zur besseren Reorganisation eines jeden Muskels unbedingt notwendig. 


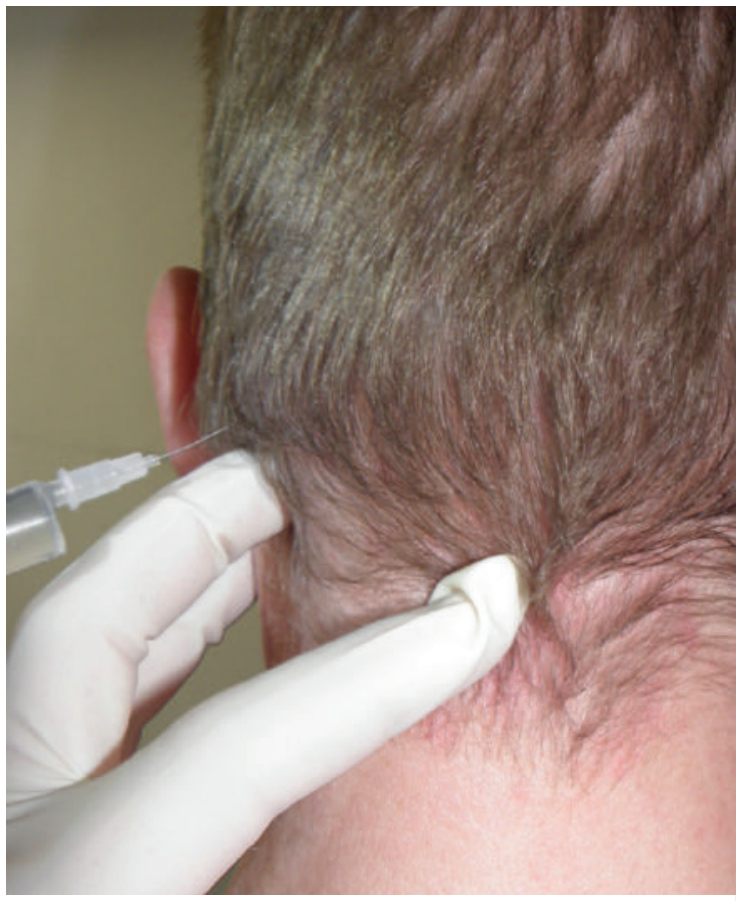

Abb.4 Injektion an den N. occipitalis major links. Foto: U. Günter

\section{Lokoregionale Neuraltherapie}

Da diese interdisziplinäre Kombinationstherapie im Alltag schwer zu organisieren und die Rezidivrate der muskulären Sekundärsymptomatik hoch ist, sind je nach Verlauf lokoregionale Injektionstechniken empfehlenswert.

Die Infiltration der Nn. occipitales major et minor kann mit [30] und ohne Sonographiekontrolle einerseits beim zervikogenen Kopfschmerz [31], aber nach auch bei allen o.g. Symptomkomplexen [32] in Bauchlage oder besser sitzend in einem Akupunkturstuhl erfolgen ( $\triangleright$ Abb. 4). In Fällen frontaler oder nuchal-lateraler Neuralgien kann ohne apparative Technik subkutan der betroffene Nerv (z. B. N. supraorbitalis oder N. accessorius) ( $>$ Abb.5) [33] infiltriert werden. Beim Rezidiv empfehle ich hier nicht die in der Datenbank Pubmed veröffentlichten Arbeiten über endoskopisch assoziierte Dekompressionen von Gesichtsnerven, CT-gestützte Injektionen an Zervikalnerven bzw. Verwendung von Kortikoiden, sondern eher eine Wiederholung in der Kombination mit einem Amid wie Mepivacain aufgrund der besseren Diffusionseigenschaften.

Je nach HRV mit erhöhtem Index nach Baevski sollte man aber immer an sympathikotone Dysregulationen bzw. neurogene Inflammationen denken [34].

Hier empfehle ich beim Nackenschmerz die monosegmentale ipsilaterale Infiltration des Ramus articularis eines zervikalen Facettengelenkes, welcher ein Ausläufer des dorsalen Astes des Spinalnerven (hier (4-8) ist, in der Technik nach Mink [35].

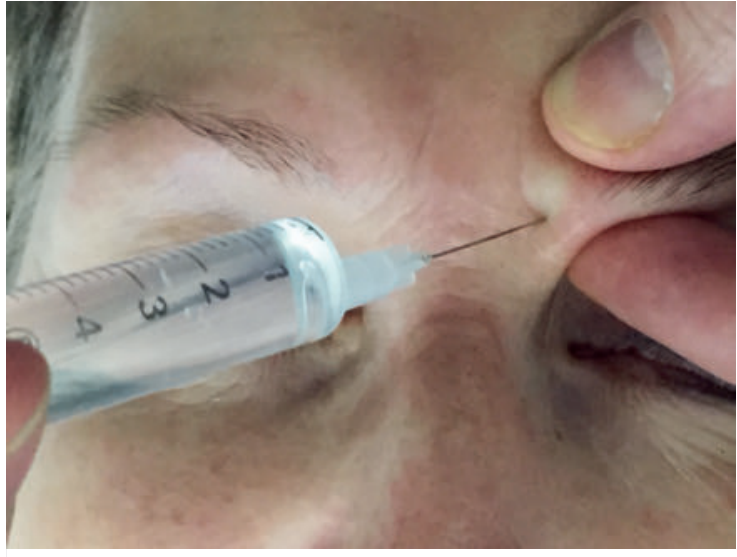

Abb. 5 Injektion an den N. supraorbitalis links. Foto: U. Günter

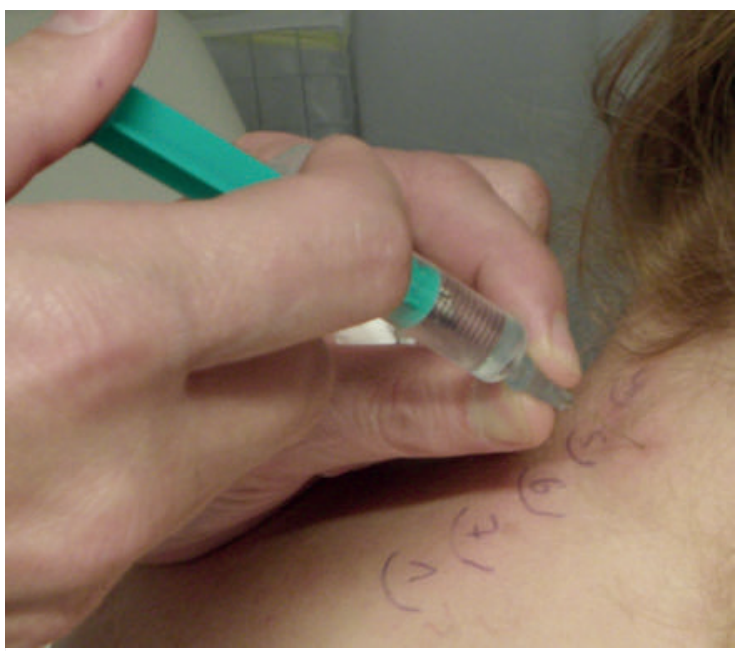

Abb. 6 Injektion nach Mink C5 rechts unter Fixation. Foto: U. Günter

Dabei nutze ich ein vibrierendes Monitoring, indem der sitzende und von der Assistenz gesicherte Patient laut zählt und ich Schwingungen bei Kanülenkontakt an der Facette spüre. Den Einstich bzw. die Markierung mit dem Finger (Dermographismus ist nach Desinfektion noch gut zu erkennen) suche ich einen halben Querfinger paramedian neben dem Mittelfinger der palpierenden Hand, welcher den oben liegenden Dorn von kaudal berührt und dadurch direkt auf dem interspinalen Band median liegt. Nach Knochenkontakt und leichtem Zurückziehen, Fixation des Konus der Kanüle mit Daumen und Zeigefinger der palpierenden Hand und einmaliger trockener Aspiration injiziere ich $2 \mathrm{ml}$ Procain $1 \%$ ( $\mathbf{A b b} .6$ ).

Beim Spannungskopfschmerz empfiehlt sich aufgrund der sympathischen perivaskulären Innervation der extrazerebralen Strukturen hier nun die Injektion an das Ganglion stellatum in der modifizierten Technik nach Dosch, welcher das Vorgehen nach Leriché, Fontaine und De Séze mit dem 


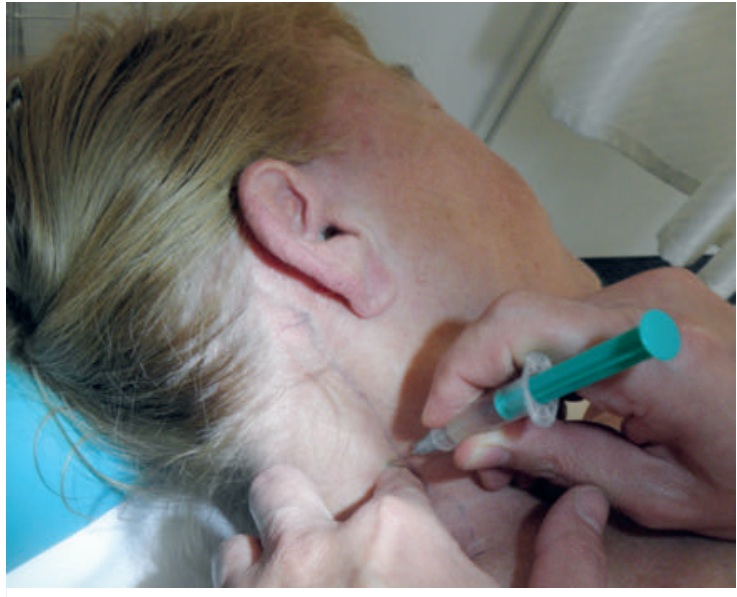

- Abb.7 Beginn der Injektion an das Ggl. stellatum rechts unter Palpation mit Kontakt am Tuberkulum caroticum vor Fixation und Aspiration. Foto: U. Günter

nach Herget verglich und verbesserte [36]. Letzteres kann auch unter Sonographie-Kontrolle erfolgen [37].

Ich empfehle zunächst die Rückenlagerung mit streng rotiertem Kopf nach kontralateral je nach subjektiver Möglichkeit und leichter Anteversion des Kopfes durch Unterpolstern eines Kissens oder einer Halbrolle. An der dorsalen Grenze des M. sternocleidomastoideus palpiere ich nach Ausmessung (zwischen Hälfte und unterem Drittel) das druckdolente Tuberculum caroticum mit der gebeugten Fingerspitze des Mittelfingers der palpierenden Hand, gebe es zur Desinfektion und Injektion mit einer mit $2 \mathrm{ml} 1$ \%igem Procain gefüllten Spritze und kurzer Kanüle $(0,6 \times 25 \mathrm{~mm})$ frei. Sollte nicht unmittelbar Knochenkontakt vorhanden sein, wiederhole ich die Palpation und Injektion je nach Schmerz und Compliance des Patienten, welcher aufgefordert wird, entweder mit den Augen zu zwinkern, der Hand zu klopfen oder mit dem Zeigen des Daumens nach „oben“ oder „unten“ sein Befinden zu dokumentieren. Bei Knochenkontakt fixiere ich den Konus wie oben beschrieben, aspiriere $2 \times$ nach Drehung der Kanüle um ca. $90 \mathrm{Grad}$ und injiziere langsam unter o. g. Monitoring ( $\bullet$ Abb. 7).

Abschließend wird der Patient aufgesetzt, sodass das Lokalanästhetikum nach kaudal zum eigentlichen Verschmelzungsort des Ganglions unter der tiefen Faszie "fließen“ und die peripheren Zeichen der temporären Ausschaltung des Sympathikus nach Horner (z. B. Ptosis oder Miosis) [38], aber auch eine generalisiertere und in der HRV sofort erkennbare Sympathikolyse bewirken kann.

\section{Störfeldtherapie}

Die tastbaren bzw. dolenten Punkte dorsal der Querfortsätze der Halswirbelkörper 1 bis 4 ( $\vee$ Abb. 8), wie sie von Adler erstmals bei Zahnerkrankungen und durch Langer zusätzlich bei Erkrankungen aller Sinnes- und Hohlorgane des Kopfes aufgearbeitet wurden, können eine diagnosti-

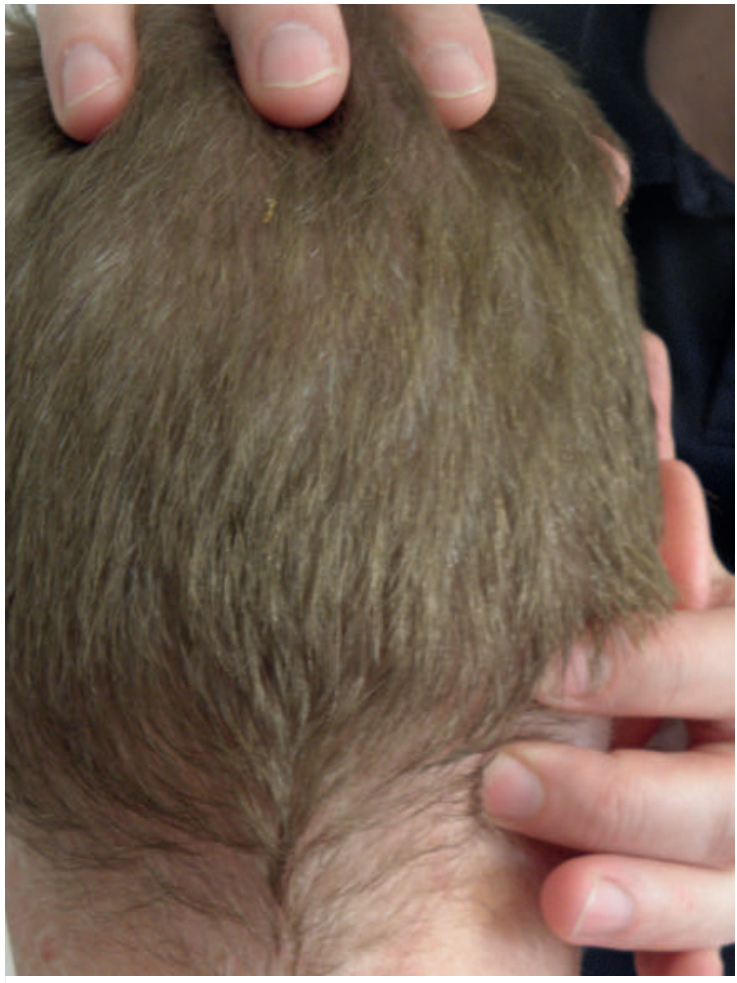

- Abb. 8 Palpation nach Adler und Langer mit Mittelfinger am Punkt 2 links unter Orientierung am Okziput mit Zeigefinger. Foto: U. Günter

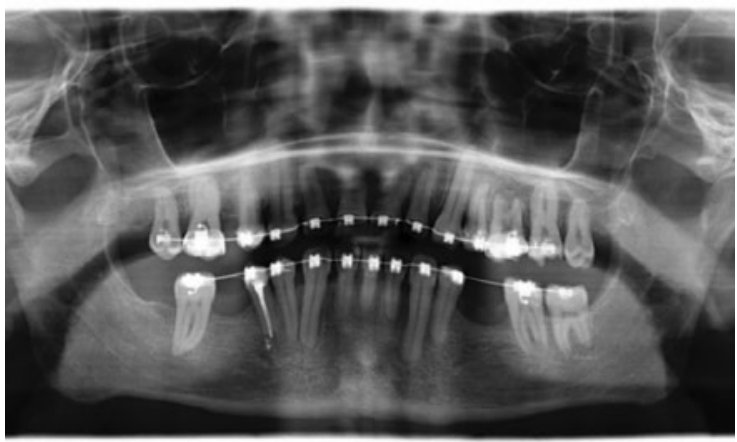

Abb. 9 Ortho-Pan-Tomogramm (OPG) bei kraniomandibulärer Dysfunktion und apikalem Herd 37 und 45. Foto: U. Günter

sche Hilfe bei der Suche nach chronisch latenten Entzündungen im Kopf-Hals-Bereich sein [39][40][41][42].

Als conditio sine qua non sehe ich bei jedem chronisch rezidivierenden Schmerz das Ortho-Pan-Tomogramm (OPT) ( Abb. 9) bzw. Tomogramme der Zähne (z. B. DVT), wie es Lechner 2008 beschrieb [43].

Seltener ist bei unauffälligem Befund ein Tomogramm der Nasennebenhöhlen ratsam. 


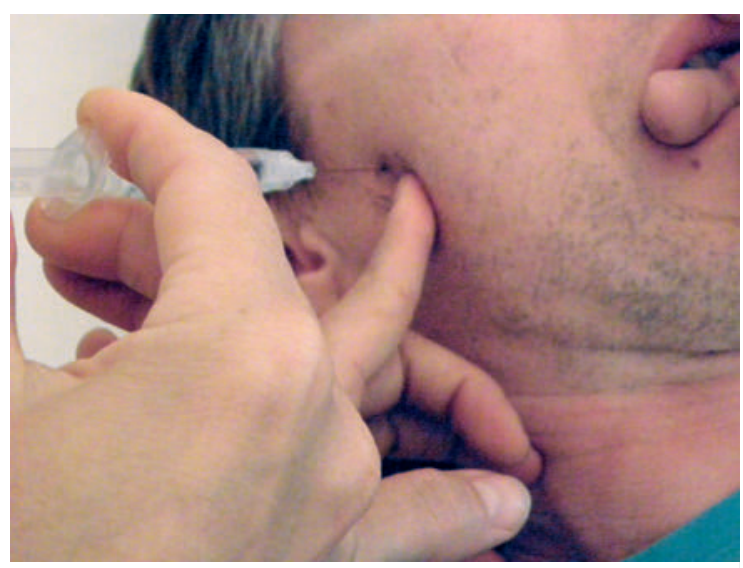

Abb. 10 Injektion an das Ganglion oticum rechts. Foto: U. Günter

Im Falle von chronisch latenten Entzündungen bzw. neuralgieinduzierenden cavitätsbildenden Osteolysen (NICO) der Kieferknochen [43] helfen prä- und postoperative Infiltrationen der vestibulären und perisinusogenen Gewebe und Nozizeptoren in den auffälligen Regionen (z. B. N. supraorbitalis, infraorbitalis, alveolaris superior sive inferior) sowie an die Ganglien des 2. und 3. Trigeminusastes (Ggll. spheno- sive pterygopalatinum et oticum) ( $\mathbf{A}$ Ab. 10). Zu jeder dieser Techniken sollte die Infiltration des Velum palatinum bzw. der peritonsillären Submukosa sowie die Palpationskontrolle der Adler-Langer-Druckpunkte gehören [41]. Ich unterscheide dabei zwischen der schmerzhaften Quantität, was der Patient spürt und der verquollenen Qualität, was nur der Untersucher fühlt und selten unmittelbar post injectionem sistiert, sondern längerfristig zu kontrollieren ist.

\section{Verlaufskontrolle}

Neben der zwischenzeitlichen Anamnese helfen die von Stamer empfohlenen somatischen Untersuchungskriterien wie der (Fehl)Stellung der Beckenknochen (Beckenverwringung), der sogenannten Schlüsselsegmente nach Wander einschl. der Blockierung im ISG bzw. SIG, der Segmente um Th10, Th4, C4 sowie der Kopfgelenke unter ggf. notwendiger Schienentherapie der von Göbel 2012 erwähnten Biss- und Kieferrelationsstörungen [44][45].

Bei zu niedriger HRV, erschöpfendem Allgemeinzustand bzw. Schmerzpersistenz veranlasse ich serologische Tests auf Zeichen einer adjuvant zu behandelnden Silent Inflammation, einem Begriff, der schon seit den 1910er-Jahren von HNO-Ärzten genutzt wird, bzw. einer sekundären Mitochondriopathie, die seit den 1960er-Jahren durch die Pathologen beschrieben wird. Hierzu empfehle ich u. a. nach [46]:

- großes Blutbild

- E-Phorese

- hochsensitives $\mathrm{CrP}$

- TNF- $\alpha$ 
- Interleukin 1

- Histamin

- CXCL10 bzw. Interferon-induziertes Protein 10

- Mannose-bindendes Lektin

- sekretorisches $\lg \mathrm{A}$

- Zink

- in vitro provozierbare Reaktionen der Lymphozyten auf Mercaptane bzw. Thioether

- CCL5 bzw. RANTES

Oft sind aber auch veränderte Metaboliten wie Vitamin $D$, Homocystein bzw. die Vitamine $B_{3}$ (Niacin), $B_{6}$ (aktiv als $\mathrm{P}-5-\mathrm{P}$ ), $\mathrm{B}_{9}$ (Folsäure) und $\mathrm{B}_{12}$ (aktiv als Holo-Transcobalamin), Coenzym Q10 sowie das von Göbel 2012 erwähnte Serotonin als Screening oder Verlaufskontrolle der homotoxikologischen oder orthomolekularen Therapie zu bestimmen [47].

Letztendlich ist der Rückgang von Schmerz anhand einer VAS, der Myogelosen bzw. Triggerpunkte, aber auch vegetativer Symptome, Fatigue- bzw. Stresszeichen in der HRV bei verbesserter Lebensqualität der inhaltliche Erfolg.

\section{Diskussion}

Wie beim Kopfschmerz ist eine Differenzialdiagnostik zur genauen Einteilung der Nackenschmerzen empfehlenswert.

Zur Begleitdiagnostik zählt aus meiner 25-jährigen Berufserfahrung neben der jungen HRV-Messung auch die altbewährte und evidente serologische Abklärung einer chronisch latenten Entzündung (Silent Inflammation) und einer zu Fatigue führenden Mitochondriopathie, welche zum großen Teil in den Leistungskatalogen der Versicherer enthalten sind.

Neben der fokussanierenden Therapie, wie es immer mehr Zahnärzte perfektionieren, sind es antientzündliche, muskelbalancierende und sympathikolytische Maßnahmen in Form von multimodaler, bio-psycho-sozialer Entspannungs-, Manual- und Neuraltherapie neben der Akupunktur eine leitlinienverbessernde Option.

Die Leitlinien der DGN und DEGAM erwägen derzeit nur Injektionsbehandlungen von Triggerpunkten bzw. bei parallelem Vorhandensein von Ohrenschmerzen und einer Zervikalneuralgie.

Die Neuraltherapie als diagnostische und therapeutische Anwendung von Lokalanästhetika ist parallel zur ärztlichen Ausbildung erlernbar, kostengünstiger und ungefährlicher als eine fehlinterpretierte Arbeit von 1970 aufgrund der Vasodilatation „allergisierte“.
Interessenkonflikte

Der Autor erklärt, dass keine wirtschaftlichen oder persönlichen Verbindungen bestehen.

Literatur

[1] Tilscher H. Das Zervikalsyndrom: konservativ-orthopädische Aspekte. Manuelle Med 2015; 53 (2): 107-110

[2] Hansen K, Schliack H. Segmentale Innervation. Stuttgart: Thieme; 1962

[3] Ashina S, Bendtsen L, Lyngberg AC et al. Prevalence of neck pain in migraine and tension-type headache: A population study. Cephalgia 2015; 35 (3): 211-219

[4] Heinz J. Topographische Anatomie der zervikalen Nervenwurzelabgänge unter spezieller Berücksichtigung der zervikalen Injektionsbehandlung [Dissertation]. Bochum: 2003

[5] Simmenroth-Nayda A. Nackenschmerzen. Die S3-Leitlinie der Deutschen Gesellschaft für Allgemeinmedizin und Familienmedizin (DEGAM). Angewandte Schmerztherapie und Palliativmedizin 2010; 2-6

[6] Göbel H. Die Kopfschmerzen. 3. Aufl. Berlin, Heidelberg: Springer; 2012

[7] Aaseth $\mathrm{K}$ et al. Pericranial tenderness in chronic tension-type headache. The Akershus population-based study of chronic headache. J Headache Pain 2014; 5 (15): 58

[8] Kristoffersen ES et al. Psychological distress, neuroticism and disability associated with secondary chronic headache - the Akershus study. J Headache Pain 2018; 19 (1): 62

[9] Seidel E]. Smartphone-Nutzung als Gefahr für die HWS? Manuelle Medizin 2015; 53 (6): 456

[10] Weinschenk S, Hollmann MW, Göllner R et al. Heidelberg University Neural Therapy Education and Research Group. Injections of local anesthetics into the pharyngeal region reduce trapezius muscle tenderness. Forsch Komplementmed 2016; 23 (2):111-116

[11] Gleditsch JM. Therapie entzündlicher Hals-, Nasen-, Ohren-, Mund- und Kieferkrankheiten durch punktuelle Lymphtherapie. Heidelberg: Springer; 1983

[12] Hülse M, Neuhuber W, Wolff HD. Die obere Halswirbelsäule. Heidelberg: Springer; 2005

[13] Di Paolo C et al. Temporomandibular disorders and headache: A retrospective analysis of 1198 patients. Pain Res Manag 2017; 3203027

[14] D'Urso A et al. Headache and temporo mandibular disorders epidemiological assessment. Minerva Stomatol 2016; 65 (2): 85-92

[15] Freese A et al. Zervikogener Kopfschmerz. Nervenheilkunde 2018; 37 (0102): 29-32

[16] Parikh P et al. Comparison of CPG's for the diagnosis, prognosis and management of non-specific neck pain: A systematic review. BMC Musculoskelet Disord 2019; 20 (1): 81

[17] Straube A. Therapie des episodischen und chronischen Kopfschmerzes vom Spannungstyp und anderer chronischer täglicher Kopfschmerzen. DGN 2015; Im Internet: www.dgn. org

[18] Stör ]. Die Studienlage zur Akupunkturwirkung bei Kopfschmerzen und Migräne. DZA 2016; 59: 25-27 
[19] Weinschenk S. Handbuch Neuraltherapie. München: Urban \&Fischer; 2010

[20] Weinschenk S. Neural therapy - A review of the therapeutic use of local anesthetics. Acupuncture and Related Therapies 2012; 1 (1): 5-9

[21] Joos S, Musselmann B, Szecsenyi J. Integration of complementary and alternative medicine into family practices in Germany: Results of a national survey. Evid Based Complement Alternat Med 2011; doi: 10.1093/ecam/nep019

[22] Zink W, Graf BM. Toxikologie der Lokalanästhetika. Anaesthesist 2003; 52: 1102-1123

[23] Hahn-Goddefroy JD, Barop H. Zur Arzneimittelsicherheit von Procain. DZA 2011; 54: 28-29

[24] Weinschenk S, Mergenthaler C, Armstrong C et al. Local anesthetics, procaine, lidocaine and mepivacaine show vasodilatation but no type 1 allergy: A double-blind, placebo-controlled study. Biomed Res Int 2017; doi: $10.1155 / 2017 / 9804693$

[25] Wander R. Neuraltherapie der Wirbelsäule und Gelenke. Ärzte Z Naturheilverfahren 1992; 33: 972-975

[26] Wander R. Kopfschmerzbehandlung mit Neuraltherapie, Manueller Medizin und Akupunktur. NHK 2014; 4: 44-46

[27] Günter U. Neuraltherapie Rückenschmerz deuten und invasive Therapie. DZA 2018; 3: 167-170

[28] Travell J et al. Pain and disability of the shoulder and arm: Treatment by intramuscular infiltration with procaine hydrochloride. JAMA 1942; 120 (6): 417-422

[29] Günter U. Komplementäre Behandlung nach VNS-Analyse: Optionen in der ganzheitlich-orientierten Sprechstunde. Naturheilkunde 2017; 5: 36

[30] Kariya K et al. Anatomical basis for simultaneous block of greater and third occipital nerves. J Anesth 2018; 32 (4):483-492

[31] Lauretti GR et al. Efficacy of the greater occipital nerve block for cervicogenic headache: Comparing classical and subcompartmental techniques. Pain Pract 2015; 15 (7): 654-661

[32] Levin M. Nerve blocks in the treatment of headache. Neurotherapeutics 2010; 7 (2): 197-203

[33] Günter U. Neuraltherapie bei nicht vertebragenen Schulterbeschwerden. zkm 2017; 3: 24-28

[34] Günter U. Entzündliche Gelenkerkrankungen neuraltherapeutisch behandeln. zkm 2018; 3: 38-41

[35] Mink E. Procaintherapie nach Huneke in der Gynäkologie. Heidelberg: Haug; 1973

[36] Dosch P. Lehrbuch der Neuraltherapie nach Huneke. 14. Aufl. Heidelberg: Haug; 1995

[37] Pu S et al. Effects of ultrasound-guided stellate ganglion block on cervical vascular blood flow study protocol for a randomized controlled trial. Trials 2018; 19 (1): 426

[38] Horner JF. Über eine Form von Ptosis. Klinische Monatsblätter für Augenheilkunde 1869; 7: 193-198

[39] Adler E. Erkrankungen durch Störfelder im Trigeminusbereich. Heidelberg: Fischer; 1973

[40] Langer H. Sonderdruck aus ZÄN 1994; 10 (35): 712-716

[41] Weinschenk S, Göllner R, Hollmann MW et al. Inter-rater reliability of neck reflex points in women with chronic neck pain. Forsch Komplementmed 2016; 23 (4): 223-229

[42] Saha FJ, Wander R. Das Störfeld als neuromodulativer Trigger. DZA 2014; 57 (2): 6-9

[43] Lechner J. Herzerkrankungen und Zahnstörfelder. EHK 2008; 57: 656-662
[44] Becke H, Wagner R, Wander R. Taschenatlas Naturkundliche Untersuchungstechniken. Stuttgart: Hippokrates; 2000

[45] Stamer N. Interdisziplinäres Biss- und Kieferrelationsmanagement part I. DZA 2018; 2: 83-86

[46] Lechner J, von Baehr V. RANTES and fibroblast growth factor 2 in jawbone cavitations: Triggers for systemic disease? Int J Gen Med 2013; 6: 277-290

[47] Von Baehr V. Die Bedeutung der Entzündung beim chronischen Schmerz. Schmerzmedizin 2018; 34 (6): 42-45

Autor

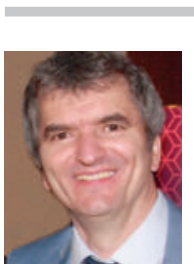

\section{Uwe Günter}

ist Facharzt für Orthopädie und Unfallchirurgie mit den Zusatzbezeichnungen Chirotherapie, Akupunktur, Sportmedizin und Physikalische Therapie. Niederlassung in eigener Praxis mit Schwerpunkt Neuraltherapie in Berlin. Dozent der Deutschen Gesellschaft für Akupunktur und Neuraltherapie.

\section{Korrespondenzadresse}

Dr. med. Uwe Günter
Siegfriedstr. 204c
10365 Berlin
E-Mail: info@dr-guenter.de

\section{Bibliografie}

DOI https://doi.org/10.1055/a-0898-2256

EHK 2019; 68: 140-147

(C) MVS Medizinverlage Stuttgart GmbH \& Co. KG ISSN 0014-0082 